Mais l'équation (4) donne

$$
-f \int_{0}^{1} \frac{d x}{x} \cdot e^{-\frac{f}{x}}=-e^{1}+\int_{0}^{1} d x \cdot e^{-\frac{f}{x}}
$$

donc, en substituant il viendra

$$
\begin{aligned}
& I=0,0789751-\frac{d f}{f}\{0,2406045-0,0789751\} \\
& I=0,0789751-\frac{\delta f}{f} \cdot 0,1616294
\end{aligned}
$$

et comme $\delta f=-2,67932 . \delta \mu$, notus avons

$$
I=0,0789751+0,30398 . \delta \mu \ldots \ldots \ldots(11)
$$

On voit par cette formule, que, en prenant $\delta \mu=\eta^{\prime} \delta$. la valeur de I devient à-peu-près un dixième; et que, en prenant $\delta \mu=-I^{\prime}$ elle s'abaisse $a$ un vingtiène. Cela suffit pour prouver que l'influence d'une erreur sur le coefficient $\mu$ $=\frac{35}{4}$ de Bouguer, peut être capable d'apporter une grande moditication sur la mesure de l'intensité $I$, qui reste à la photosphère du Soleil, après avoir termiıé l'atmosphère absorbante qui l'entoure.

Turin le 28 mai 1852 .

\title{
Jean Plana.
}

\section{Ueber die Säcularänderungen der Präcession im British Assoc. Catalogue.}

Ich erlaube mir hier eine kleine Bemerkung, die Berechnung der Säcularänderungen der Präcession betreffend. Sind $p$ und $p^{\prime}$ die einjăhrigen Prïcessionen in Rectascension und Declination, so ist bekanntlich $p=m+n \operatorname{tg} \delta \sin \alpha, p^{\prime}=n \cos \alpha$. Die Werthe $\alpha, \delta, m$ und $n$ gelten aber nur für eine bestimmte Epoche, nit ihnen ändern sich auch $p$ und $p^{\prime}$ and zwar il einem Jahre um

$$
\begin{aligned}
& d p=d m+d n \operatorname{tg} \delta \sin \alpha+n \operatorname{tg} \delta \cos \alpha \sin 1^{\prime \prime} d \alpha \\
& +n \sin \alpha \sec \delta^{2} \cdot \sin 1^{\prime \prime} d \delta, \\
& d p^{\prime}=d n \ln \alpha-n \sin \alpha \sin 1^{\prime \prime} d \alpha,
\end{aligned}
$$

weun $d m, d n$, $1 x$ und d die einjährigen Aenderungen jener Grössen bedeuten. Aber $d \alpha=p$, d $\delta=p^{\prime}$, und damn

$$
d p=d m+d n \operatorname{tg} \delta \sin \alpha+p p^{\prime} \operatorname{tg} \delta \sin 1^{\prime \prime}+p^{\prime 2} \sec d^{2} \operatorname{tg} \alpha \sin 1^{\prime \prime}
$$$$
d p^{\prime}=d n \cos \alpha-n p \sin x \sin 1^{\prime \prime} \text {. }
$$

Die Werthe derjenigen Glieder unn, welche $d m$ und $d n$ enthalten, sind häutig für so gering gehalten, dass sie ganz vernachlässigt werden können, oder es sind geradezu $d m$ und $d n=0$ gesetzt, während maı doch bei der gewöhnlichen Mathode der Reduction mit Hülfe dè für die Mitte da.r heiden Zeiten geltenden Präcession, auf die Aenderung von in und $n$ sehr wohl Rücksicht nimmt. Die Voraussetzung $d m$ und $d n$ $=0$ ist z. B. bei der Berechnung der Säcularänderungen in dem British Assoc. Cat. gemacht (Preface, p. 39). Sie sind daselbst bis auf 4 Stellen in AR.. Zeit. und 3 Stellen in Decl. angesetzt und daber die Vernachlässigung der erwähnten Glieder nur dann gestattet, wenn ihr Betrag den Werth einer Einheit dieser Stellen nicht erreicht. Es ist nun aber nach Bessel

$$
\begin{aligned}
\text { I's } 100 d_{m} & =+0,0020576, \quad \text { 1' } 100 d_{n}=-0,0006468, \\
100 d n & =-0,00970204
\end{aligned}
$$

und danit der Betrag, welcher an die Angalıen des Katalngs noch anzubringen ist $=+0,0020576-0,0006468 \operatorname{tg} \delta \sin \alpha$ in AR. und +0,00970204 ros $\alpha$ in Nordpolardistanz. Die fulgende erste: Tafel giebt nit den Argumenten $\alpha$ und $\delta$ den Werth -0,0006468 $\lg \delta \sin \alpha$, der dan!n noch mit dem richtigen Zeichen zu $+0,00206$ hinzuzufügen ist. Die Zeichen gelten für nördliche und sürliche Declinationen und für die ersten 12 Stunden der AR., sind also für $12^{\mathrm{h}}$ bis $23^{\mathrm{h}}$ die entgegengesetzten. Die Zahlen bedeuten Einheiteu der jten Stelle. Die zweite Tafel giebt mit dem Argument $\alpha$ die Correction für die Nordpolardistanz in Eimheiten der 3 ten Stelle. Man sieht daraus z. B., dass die Correction der Säcularianderungen in AR. im Maximo, d. i. für $18^{\text {h }}$ lrei nördl. und für $6^{\text {h }}$ bei sildlichen Declinationen

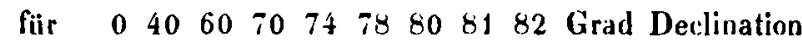
beträgt $+21 \quad 26 \quad 32 \quad 3843 \quad 51 \quad 57 \quad 6167$ Eiulseiten d. 4. Stelle.

Sobald aber die eigene Bewegung des Sterns merklich wird, ist, besonders für hohe Declinationen, die Genauigkeit bis anf eine Einheit resp. der $4^{\text {ten }}$ und $3^{\text {ten }}$ Stelle nur illusorisch, indem der zweite und die folgenden Differentialquotienten Glieder entbalten, die von der eigenen Bewegung abhängig sind, und doch noch zur reinen Reduction gehören, so dass der erst wach ihrer Anbringung sich noch zeigende Unterschied der Wirkung der eigenen Bewegung allein zugeschrieben werden kann. Statt nämlich $p$ und $p^{\prime}$ für $d \alpha$ und $d \delta$ zu setzen, hätten wir $p+\mu$ und $p^{\prime}+\mu^{\prime}$ nchmen müssen, wenn $\mu$ und $\mu$ die eigenen Bewegungeu in AR. und Decl. bezeichnen. Dadurch ist dem $d p$ hinzuzufügen $\mu p^{\prime} \operatorname{tg} d \sin 1^{n}$ $+\mu^{\prime} n \sec \delta^{2} \sin \alpha \sin 1^{\prime \prime}$ und dem $d p^{\prime} n o c h-\mu n \sin \alpha \sin 1^{\prime \prime}$. (S. Tab. Reg. p. XIV. und Argelander: Positiones mediae p. XII.) Das Verfahren, zur Ableitung der Säcularänderungen sich aweier Präcessionen zu bedienen, die auf zu ver- 
schiedenen Zeiten gemachten wirklicben Beobachtungen heru. hen, ist hiernach jedenfalls das bequemste und genaueste, beiden Beobachtungsepochen als gleichförmig angesehen werrorausgesetzt uatürlich, dess die Aanderung innęrhalb der den kann.

\section{Tafel I.}

Nürdliche Declination.

\begin{tabular}{|c|c|c|c|c|c|c|c|c|c|c|c|c|c|c|c|c|c|c|}
\hline & $0^{n}$ & $10^{\circ}$ & $20^{\circ}$ & $30^{\circ}$ & $40^{\circ}$ & $50^{\circ}$ & $55^{\circ}$ & $60^{\circ}$ & $65^{\prime \prime}$ & $70^{\circ}$ & $72^{\prime \prime}$ & $74^{\circ}$ & $76^{\circ}$ & $78^{\circ}$ & $80^{\circ}$ & $81^{\circ}$ & $82^{\circ}$ & \\
\hline & & - & - & - & - & - & - & - & - & - & - & - & - & - & - & - & - & \\
\hline $0^{\mathrm{h}} \quad 0^{\mathrm{m}}$ & 0 & 0 & 0 & 0 & 0 & 0 & 0 & o & 0 & 0 & $\overline{0}$ & 0 & 0 & O & 0 & 0 & 0 & $12^{h} 0^{\pi}$ \\
\hline 20 & 0 & 1 & 2 & 3 & b & 7 & 8 & 10 & 12 & 15 & 17 & 20 & 23 & 27 & 32 & 36 & 40 & 40 \\
\hline 40 & 0 & 2 & 4 & 6 & 9 & 13 & 16 & 19 & 24 & 31 & 35 & 39 & 45 & 53 & 64 & 71 & 80 & 20 \\
\hline 10 & 0 & 3 & 6 & 10 & 14 & 20 & 24 & 29 & 36 & 46 & 52 & 58 & 67 & 79 & 95 & 106 & 119 & 110 \\
\hline 20 & 0 & 4 & 8 & 13 & 19 & 26 & 32 & 38 & 47 & 61 & 68 & 77 & 89 & 104 & 125 & 140 & 157 & 40 \\
\hline 40 & 0 & 5 & 10 & 16 & 23 & 33 & 39 & 47 & 59 & 75 & 84 & 95 & 110 & 129 & 155 & 173 & 194 & 20 \\
\hline 0 & 0 & 6 & 12 & 19 & 27 & 39 & 46 & 56 & 69 & 89 & 100 & 113 & 130 & 152 & 183 & 204 & 230 & 100 \\
\hline 20 & 0 & 7 & 14 & 21 & 31 & 44 & 53 & 64 & 80 & 102 & 114 & 129 & 149 & 175 & 210 & 234 & 264 & 40 \\
\hline 40 & 0 & 7 & 15 & 24 & 35 & 50 & 59 & 72 & 89 & 114 & 128 & 145 & 167 & 196 & 236 & 262 & 296 & 20 \\
\hline 0 & 0 & 8 & 17 & 26 & 38 & 53 & 65 & 79 & 98 & 126 & 141 & 159 & 183 & 215 & 259 & 289 & 325 & 90 \\
\hline 20 & 0 & 9 & 18 & 29 & 42 & 59 & 71 & 86 & 106 & 136 & 152 & 173 & 199 & 233 & 281 & 313 & 353 & 40 \\
\hline 40 & 0 & 9 & 19 & 31 & 44 & 63 & 76 & 92 & 113 & 146 & 163 & 185 & 212 & 249 & 300 & 335 & 377 & 20 \\
\hline 40 & 0 & 10 & 20 & 32 & 47 & 67 & 80 & 97 & 120 & 154 & 172 & 195 & 225 & 263 & 318 & 354 & 399 & \\
\hline 20 & 0 & 10 & 21 & 34 & 49 & 70 & 84 & 102 & 126 & 161 & 180 & 204 & 235 & 276 & 332 & 370 & 417 & 40 \\
\hline 40 & 0 & 11 & 22 & 35 & 51 & 72 & 87 & 105 & 130 & 167 & 187 & 212 & 244 & 286 & 345 & 384 & 433 & 20 \\
\hline 50 & 0 & 11 & 23 & 36 & 52 & 74 & 89 & 108 & 134 & 172 & 192 & 218 & 25 & 294 & 354 & 394 & 444 & 70 \\
\hline 20 & 0 & 11 & 23 & 37 & 54 & 76 & 91 & 110 & 137 & 175 & 196 & 222 & 255 & 300 & 361 & 402 & 453 & 40 \\
\hline 40 & 0 & 11 & 23 & 37 & 54 & 77 & 92 & 112 & 138 & 177 & 198 & 225 & 25 & 303 & 365 & 4.07 & 458 & 20 \\
\hline 0 & 0 & 11 & 24 & 37 & 54 & 77 & 92 & 112 & 139 & 178 & 199 & 226 & 259 & 304 & 367 & 408 & 460 & $\begin{array}{ll}6 & 0 \\
\end{array}$ \\
\hline & & + & + & + & + & + & + & + & + & + & + & + & + & + & + & + & + & \\
\hline & 0 & $10^{\circ}$ & $20^{\prime \prime}$ & $30^{\circ}$ & $40^{\circ}$ & $50^{\circ}$ & $55^{\circ}$ & $60^{\prime \prime}$ & $65^{\circ \prime}$ & $70^{\prime \prime}$ & $72^{\circ}$ & 14 & $76^{\circ}$ & 18 & $80^{\circ}$ & 81 & $82^{\circ}$ & \\
\hline
\end{tabular}

Südliche Declination.

Wien 1852. Mai 21.

Tafel 11.

\begin{tabular}{|c|c|c|c|c|c|c|}
\hline $0^{h}$ & und & $2 q^{h}$ & $+10-$ & $12^{\mathrm{h}}$ & und & $12^{\mathrm{h}}$ \\
\hline 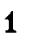 & ", & 23 & $+9-$ & 11 & " & 13 \\
\hline 2 & " & 22 & $+8-$ & 10 & " & 14 \\
\hline 3 & ", & 21 & $+7-$ & 9 & " & 15 \\
\hline 4 & ", & 20 & $+5 \ldots$ & 8 & " & 16 \\
\hline 5 & ", & 19 & $+3-$ & 7 & $"$ & 17 \\
\hline 6 & $"$ & 18 & 0 & 6 & ", & 18 \\
\hline
\end{tabular}

Beobachtungen des Cometen I. 1852 zu Königsberg.

Den in Mai zu Marseille und Altona entreckten Cometen habe ich in den Tagen Mai 21 bis 24 viermal beobachtet, kann jedoch für jetzt nur drei dieser Boobachtungen mittheilen, da der Vergleichstern für Mai 22 nirgends bestimmt ist. Der eingetretene Mondschein und die hellen Sommernächte verhinderten die weitere Verfolgung dieses lichtschwachen Cometen. Die gefundenen scheinbaren Oerter desselben sind folgende:

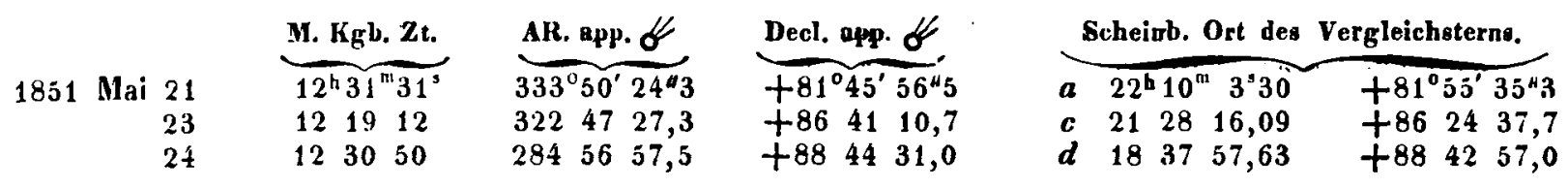

